\title{
Efficient interface conditions for the semi-vectorial finite-difference beam propagation method
}

\author{
F. WIJNANDS ${ }^{1,3}$, T. RASMUSSEN ${ }^{2}$, H. J. W. M. HOEKSTRA ${ }^{1}$, \\ J. H. POVLSEN ${ }^{2}$, A. BJARKLEV ${ }^{2}$, R. M. DE RIDDER ${ }^{1}$ \\ ${ }^{1}$ MESA Research Institute, University of Twente, P.O. Box 217, 7500 AE \\ Enschede, The Netherlands \\ ${ }^{2}$ Center for Broadband Telecommunications, Electromagnetics Institute, \\ Technical University of Denmark, DK-2800 Lyngby, Denmark \\ ${ }^{3}$ Present address: Condensed Matter Theory Group, The Blackett Laboratory, \\ Imperial College, London SW7 2BZ, UK
}

Received 2 December 1994; revised 9 May; accepted 15 May 1995

Efficient interface conditions (EICs) are derived for the propagation equation using the slowly varying envelope approximation for the dominant electric field component. At the interface between two different media, the two lateral second derivatives in the discretized propagation equation are adapted such that the discretized modal field equation is correct up to second order in the lateral grid spacing. Since the error term is then of the order of the lateral grid spacing, our EICs are first-order EICs. These interface conditions are compared with well-known zero-order EICs derived by Stern and Kim and Ramaswamy. It is shown that the first-order EICs yield faster convergence to the exact effective index value as the lateral grid spacing is decreased than do the zero-order EICs. It turns out that our EICs are very much like those derived by Vassallo. Using essentially the same method, he derived EICs of second and first order for the field component respectively parallel and perpendicular, to the interface. Hence the accuracy of his EICs is one order higher for the field component parallel to the interface, although it introduces an extra asymmetry in the propagation matrix.

\section{Introduction}

Beam propagation methods (BPMs) are very powerful for simulation of the propagation of light in structures which cannot be treated analytically. Two frequently used BPMs are the Fourier transform BPM (FTBPM) [1-3] and the finite-difference BPM (FDBPM) [4-7]. Special care has to be taken at grid points located near an interface between two different media. In the FTBPM the continuity relations at an interface cannot be treated properly. For the FDBPM these continuity relations can be taken into account by what will be called efficient interface conditions (EICs). For slab waveguides, EICs have been derived for both the TE case and the TM case by Hoekstra et al. [8]. Owing to the EICs, the discretized modal field equation (see Equation 16) is made correct up to second order in the lateral coordinate. Since the 
error term is then of the order of the lateral grid spacing, the EICs are said to be first order. Note that for grid points far from the interface, this first-order accuracy is automatically satisfied if the second derivative is approximated by a three-point operator in the discretized equation.

In three dimensions, zero-order EICs have been derived by Stern [9] in a semivectorial formulation for both quasi-TE and quasi-TM modes. The procedure consists of adapting the second-order derivative in the direction of the step in the refractive index. In that study the interface was located midway between two grid points using a uniform grid. Kim and Ramaswamy derived EICs using a similar approach for a general position of the interface with respect to the grid points, using a nonuniform grid [10]. It is the aim of this paper to present first-order EICs in a semivectorial formulation, and to compare our method with the zeroorder methods described in [9] and [10].

In order to compare these methods, modal fields and their corresponding effective indices are calculated using the propagation equation. Following an idea by Yevick and Hermansson [13], the field is propagated along an imaginary propagation axis (throughout this paper, the propagation direction is along the $z$-axis). In doing this, for a modal field with propagation constant $\beta$, a phase change $\mathrm{e}^{-1 \beta z}$ becomes an amplification factor $\mathrm{e}^{+\beta z}$. They used the FTBPM. This idea has been applied to the FDBPM using the power method for two-dimensional cross-sections [14-16] or the inverse iteration method (IIM) for one-dimensional cross-sections [17-19]. In this paper we apply the IIM to two-dimensional cross-sections, and the matrix equation will be solved using the alternating direction implicit (ADI) method [20-22]. Our study of the suitability of the $\mathrm{ADI}$ method for solving the matrix equation will be published elsewhere. Here the emphasis will be on the EICs. If needed, the method is suitable for arbitrary refractive index profiles. The profile is approximated by a staircase structure with interfaces parallel to the three Cartesian axes.

One of the referees drew our attention to two papers by Vassallo $[11,12]$, in which he also derives EICs in a semivectorial formulation, using essentially the same arguments. In the first paper [11], EICs of second (first) order are derived for the electric field parallel (perpendicular) to the interface, respectively. The second paper [12] deals with the corners of the waveguide, where the EICs break down. Our results have been derived independently, but the two formulations of the EICs turn out almost to coincide. A global (not numerical) comparison of our EICs and the EICs obtained in $[11,12]$ will be given in the Results section.

The paper is organized as follows. In Section 2 the propagation equation is derived and the mode finding process is discussed briefly. In Section 3 the EICs are derived. Also, the EICs derived by Kim and Ramaswamy [10] are reformulated in a uniform grid fashion and then compared to the EICs described by Stern [9]. The numerical results for some two-dimensional rectangular structures are presented in Section 3. A discussion of the results follows in Section 4.

\section{Theory}

The problem is worked out in terms of the electric field $E$ for the dominant field component: a quasi-TE mode (dominant field component along $x$ ) and a quasi-TM mode (dominant field component along $y$ ). Rewriting the Maxwell equations yields

$$
\left(\nabla^{2}+n^{2} k^{2}\right) \boldsymbol{E}+\nabla\left[\boldsymbol{E} \cdot \nabla \ln \left(n^{2}\right)\right]=0
$$

\subsection{Derivation of the propagation equation}

A scalar field distribution $E(x, y, z)$ can be written, according to the slowly varying envelope 
approximation (SVEA), as

$$
E(x, y, z) \equiv \psi(x, y, z) \mathrm{e}^{-\mathrm{i} k_{0} n_{0} z}
$$

where $n_{0}$ is a suitably chosen mean refractive index. In the particular case of quasi-TE and quasi-TM, substitution of Equation 2 into Equation 1 leads to

$$
\begin{gathered}
\frac{\partial^{2} \psi}{\partial z^{2}}-2 \mathrm{i} k_{0} n_{0} \frac{\partial \psi}{\partial z}+\left[\frac{\partial^{2}}{\partial x^{2}}+\frac{\partial^{2}}{\partial y^{2}}+k_{0}^{2}\left(n^{2}-n_{0}^{2}\right)\right] \psi \\
+\frac{\partial \psi}{\partial x} \frac{\partial \ln \left(n^{2}\right)}{\partial x}+\psi \frac{\partial^{2} \ln \left(n^{2}\right)}{\partial x^{2}}=0 \quad \text { (quasi-TE) } \\
\frac{\partial^{2} \psi}{\partial z^{2}}-2 \mathrm{i} k_{0} n_{0} \frac{\partial \psi}{\partial z}+\left[\frac{\partial^{2}}{\partial x^{2}}+\frac{\partial^{2}}{\partial y^{2}}+k_{0}^{2}\left(n^{2}-n_{0}^{2}\right)\right] \psi \\
+\frac{\partial \psi}{\partial y} \frac{\partial \ln \left(n^{2}\right)}{\partial y}+\psi \frac{\partial^{2} \ln \left(n^{2}\right)}{\partial y^{2}}=0 \quad \text { (quasi-TM) }
\end{gathered}
$$

Now $\psi$ is assumed to vary slowly as a function of $z$ (see Equation 2). Hence the terms $\partial^{2} \psi / \partial z^{2}$ in Equations 3 and 4 can be neglected.

Additionally, the terms containing $\partial \ln \left(n^{2}\right) / \partial x$ and $\partial \ln \left(n^{2}\right) / \partial y$ are nonzero only near interfaces. For the moment these terms are neglected. They will be taken into account in Section 3 when discussing the EICs. The field equation to be solved is then

$$
-2 \mathrm{i} k_{0} n_{0} \frac{\partial \psi}{\partial z}+\left[\frac{\partial^{2}}{\partial x^{2}}+\frac{\partial^{2}}{\partial y^{2}}+k_{0}^{2}\left(n^{2}-n_{0}^{2}\right)\right] \psi=0
$$

Now discretize the field, $\psi_{k}^{s}=\psi_{m, j}^{s} \equiv \psi(s \Delta z, m \Delta x, j \Delta y), k \equiv m+(j-1) N_{x} ; 1 \leq m \leq N_{x}$, $1 \leq j \leq N_{y}, 1 \leq k \leq N$ with $N=N_{x} N_{y}$. Here $N_{x}$ and $N_{y}$ are the number of grid points in $x$ and $\boldsymbol{y}$-directions, respectively. In some cases the one-dimensional description of the field (using one subscript) is more convenient. In other cases the two-dimensional description is more appropriate. Introduce the vector $\Psi^{s},\left(\Psi^{s}\right)_{k} \equiv \psi_{k}^{s}$. Then Equation 5 is integrated for the discretized field, using the Crank-Nicholson scheme. The result is [5]

$$
\left[\boldsymbol{M}-\mathrm{i} \frac{4 k_{0} n_{0}}{\Delta z} \mathbf{1}\right] \Psi^{s+1}=\left[\boldsymbol{M}+\mathrm{i} \frac{4 k_{0} n_{0}}{\Delta z} 1\right] \Psi^{s}
$$

with 1 being the identity matrix. Here $M$ is a five-band matrix with

$$
\left.\begin{array}{rl}
\boldsymbol{M}_{k, k} & =\frac{-2}{(\Delta x)^{2}}+\frac{-2}{(\Delta y)^{2}}+k_{0}^{2}\left(n_{m, j}^{2}-n_{0}^{2}\right) \\
\boldsymbol{M}_{k, k \pm 1} & =\frac{1}{(\Delta x)^{2}} \\
\boldsymbol{M}_{k, k \pm N_{x}} & =\frac{1}{(\Delta y)^{2}} \\
\boldsymbol{M}_{k, p} & =0 \text { elsewhere }
\end{array}\right\} 1 \leq k \leq N
$$

In case the grid point is at a boundary of the computational window, for real axis propagation, transparent boundary conditions introduced by Hadley are used [23]. In the inverse iteration method, the propagation is along a complex propagation axis (see Section 2.2). Then an adapted form of Hadley's transparent boundary conditions is used, which is illustrated for 
one boundary:

$$
\psi_{m, N_{y}}^{s+1}=\psi_{m, N_{y}-1}^{s+1} \times \max \left(1, \frac{\left|\psi_{m, N_{y}-1}^{s+1}\right|}{\left|\psi_{m, N_{y}-2}^{s+1}\right|}\right)
$$

\subsection{The mode finding algorithm}

In this subsection we discuss how to find eigenvalues and corresponding eigenvectors of the matrix $\boldsymbol{M}$ defined in Equation 6. For this purpose the same propagation equation (6) can be used, but now the propagation is along a complex (purely imaginary in the case of a lossless structure) $z$-axis. By choosing the subsequent values of $\Delta z$ suitably, the field eventually converges to a modal field. These suitable values of $\Delta z$ can be chosen according to the inverse iteration method (IIM) [24], as follows. We denote the sth iterated vector by $\Psi^{s}$ and the corresponding approximate eigenvalue by $\tau_{s} \equiv \mathrm{i}\left(4 k_{0} n_{0} / \Delta z\right)$ (e.g. by comparing Equations 6 and 7). The matrix equation is

$$
\left[\boldsymbol{M}-\tau_{s} \mathbf{1}\right] \boldsymbol{w}^{s+1}=\left[-\boldsymbol{M}-\tau_{s} \mathbf{1}\right] \Psi^{s}
$$

A new approximation of the eigenvalue is calculated by the Rayleigh quotient

$$
\tau_{s}=\frac{\left\langle\boldsymbol{M} \Psi^{s}, \Psi^{s}\right\rangle}{\left\langle\Psi^{s}, \Psi^{s}\right\rangle}
$$

with inner product $\langle\Omega, \Lambda\rangle \equiv \sum_{j=1}^{N}\left[\Omega_{j}\left(\Lambda_{j}\right)^{*}\right]$. Finally, $\Psi^{s+1}=\boldsymbol{w}^{s+1} /\left|\boldsymbol{w}^{s+1}\right|$. To solve the matrix equation problem (7), the alternating direction implicit (ADI) method [20-22] is used. The ADI method is a very efficient method making use of the special form of the matrix equation (7). Following the analysis given in [20], the ADI equations for Equation 7 can be written as

$$
\begin{aligned}
{\left[\boldsymbol{M}_{x}-\tau_{s} \mathbf{1}\right] \boldsymbol{w}^{s+1 / 2} } & =\left[-\boldsymbol{M}_{y}-\tau_{s} \mathbf{1}\right] \Psi^{s} \\
{\left[\boldsymbol{M}_{y}-\tau_{s} \mathbf{1}\right] \boldsymbol{w}^{s+1} } & =\left[-\boldsymbol{M}_{\boldsymbol{x}}-\tau_{s} \mathbf{1}\right] \boldsymbol{w}^{s+1 / 2}
\end{aligned}
$$

with

$$
\begin{array}{ll}
M_{x_{k, k}}=\frac{-2}{(\Delta x)^{2}}+\frac{k_{0}^{2}}{2}\left(n_{m, j}^{2}-n_{0}^{2}\right) & M_{x_{k, k \pm 1}}=\frac{1}{(\Delta x)^{2}} \quad M_{x_{k, p}}=0 \text { elsewhere } \\
M_{y_{k, k}}=\frac{-2}{(\Delta y)^{2}}+\frac{k_{0}^{2}}{2}\left(n_{m, j}^{2}-n_{0}^{2}\right) & M_{y_{k, k \pm N_{x}}}=\frac{1}{(\Delta y)^{2}} \quad M_{y_{k p}}=0 \text { elsewhere }
\end{array}
$$

Here $\boldsymbol{w}^{s+1 / 2}$ is an intermediate field. Furthermore, $k=m+(j-1) N_{x} ; 1 \leq m \leq N, 1 \leq j \leq N$. Note that $\boldsymbol{M}=\boldsymbol{M}_{x}+\boldsymbol{M}_{\boldsymbol{y}}$. For grid points at a boundary, the adapted form of the transparent boundary conditions described in Section 2.1 is used.

In the mode search using the IIM, the value of $|\Delta z|$ might be quite large. Owing to the inaccuracy in the ADI method as a function of $|\Delta z|$ (for the Laplace equation the error is of order $\sim O\left((\Delta z)^{3}+\Delta z\left(\Delta^{3}\right)\right)$ if $\left.\Delta x=\Delta y \equiv \Delta[20]\right)$, the mode and its eigenvalue will not be determined accurately. Therefore a refined $\mathrm{ADI}$ method is used, which consists of the following.

Recalling that $\tau_{s}=\mathrm{i}\left(4 k_{0} n_{0} / \Delta z\right)$, the ADI method will be more accurate if we divide the propagation along $\Delta z$ into $n$ steps of $\Delta z / n$. In the IIM scheme, each new approximation of the eigenvalue $\tau_{s}$ yields a new value for $\Delta z$. Inside the propagation part, the calculation is 
done $n$ times with a constant step of $\Delta z / n$, instead of once with a step of $\Delta z$. One could argue that, as a consequence of this procedure, the determination of $\Delta z$ according to Equation 8 becomes meaningless, since we are subdividing this value afterwards. Notice that the value of $\Delta z$ according to Equation 8 determines which matrix equation problem is to be solved. The only purpose of the refinement is to solve this matrix equation problem more accurately.

\section{Efficient interface conditions}

\subsection{First-order EICs}

When a step in the refractive index occurs, the continuity relations at the interface should be satisfied. We will require these continuity relations to be satisfied up to second order in the lateral step sizes $\Delta x$ and $\Delta y$ (hence the error term is of order $\Delta x, \Delta y$ ) and follow the approach of [8]. Only interfaces parallel or perpendicular to the dominant field component are considered. Suppose the normal of the interface to be parallel to $x$. Let the interface have distance $\epsilon \Delta x(0 \leq \epsilon<1)$ to grid point $(i, j)$ and distance $(1-\epsilon) \Delta x$ to grid point $(i+1, j)$, as sketched in Fig. 1. In the following, the subscript $l(r)$ refers to infinitesimally left (right) of the interface.

\section{Case A: $\psi$ parallel to interface}

In this case, (i) $\psi$ is continuous, $\psi_{l}=\psi_{r}$; (ii) $\partial \psi / \partial x$ is continuous, $(\partial \psi / \partial x)_{l}=(\partial \psi / \partial x)_{r}$; (iii) $\partial^{2} \psi / \partial y^{2}$ is continuous, $\left(\partial^{2} \psi / \partial y^{2}\right)_{l}=\left(\partial^{2} \psi / \partial y^{2}\right)_{r}$. The last continuity relation can be understood as follows. Assume that the field may be written as a product $\psi(x, y)=a(x) b(y)$. Then $\partial^{2} \psi(x, y) / \partial y^{2}=a(x)\left[\partial^{2} b(y) / \partial y^{2}\right]$. The functions $a(x)$ and $\partial^{2} b(y) / \partial y^{2}$ are both continuous at the interface, hence $\left(\partial^{2} \psi / \partial y^{2}\right)_{l}=\left(\partial^{2} \psi / \partial y^{2}\right)_{r}$. This continuity relation is strictly valid only for an infinitely extended interface, hence near corners the relation is not strictly valid. Now $\left(\partial^{2} \psi / \partial x^{2}\right)_{r}$ and $\left(\partial^{2} \psi / \partial x^{2}\right)_{l}$ are related by comparing the modal field equations at the right and the left of the interface:

$$
\left(\frac{\partial^{2} \psi}{\partial x^{2}}\right)_{r}=k_{0}^{2}\left[n_{e}^{2}-n_{r}^{2}\right] \psi_{l}-\left(\frac{\partial^{2} \psi}{\partial y^{2}}\right)_{r} \quad\left(\frac{\partial^{2} \psi}{\partial x^{2}}\right)_{l}=k_{0}^{2}\left[n_{e}^{2}-n_{l}^{2}\right] \psi_{l}-\left(\frac{\partial^{2} \psi}{\partial y^{2}}\right)_{l}
$$

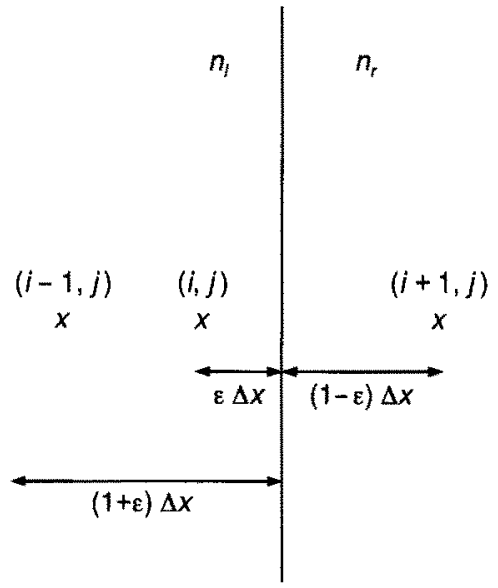

Figure 1 General position of an interface parallel to the $y$-axis between two media of refraclive index $n_{i}$ and $n_{r}$ at the left and the right of the interface, respectively. The interface has distance $\epsilon \Delta x(0 \leq \epsilon<1)$ to grid point $(i, j)$ and distance $(1-\epsilon) \Delta x$ to grid point $(i+1, j)$. 
Here $n_{e}$ is the effective index of the mode; $n_{r}, n_{l}$ are refractive indices at the right and left of the interface, respectively. From relation (iii), $\left(\partial^{2} \psi / \partial x^{2}\right)_{r}=\left(\partial^{2} \psi / \partial x^{2}\right)_{l}+k_{0}^{2}\left[n_{l}^{2}-n_{r}^{2}\right] \psi_{l}$.

Our approach comes down to adapting the second derivative

$$
\frac{\partial^{2} \psi_{i, j}}{\partial x^{2}}=\frac{L_{1} \psi_{i-1, j}+L_{2} \psi_{i, j}+L_{3} \psi_{i+1, j}}{(\Delta x)^{2}}+O(\Delta x)
$$

such that the continuity relations (i)-(iii) are satisfied. Since the error term is then of order $O(\Delta x)$, the method is a first-order method. Away from the interface, the modal field equations are already of first order for the standard values $L_{1}=L_{3}=1, L_{2}=-2$. Up to second order the Taylor expansions read

$$
\begin{aligned}
\psi_{i+1, j} & =K \psi_{l}+(1-\epsilon) \Delta x\left(\frac{\partial \psi}{\partial x}\right)_{r}+\frac{(1-\epsilon)^{2}}{2}(\Delta x)^{2}\left(\frac{\partial^{2} \psi}{\partial x^{2}}\right)_{r} \\
\psi_{i, j} & =\psi_{l}-\epsilon \Delta x\left(\frac{\partial \psi}{\partial x}\right)_{l}+\frac{\epsilon^{2}}{2}(\Delta x)^{2}\left(\frac{\partial^{2} \psi}{\partial x^{2}}\right)_{l} \\
\psi_{i-1, j} & =\psi_{l}-(1+\epsilon) \Delta x\left(\frac{\partial \psi}{\partial x}\right)_{l}+\frac{(1+\epsilon)^{2}}{2}(\Delta x)^{2}\left(\frac{\partial^{2} \psi}{\partial x^{2}}\right)_{l}
\end{aligned}
$$

From continuity relation (i), $K=1$. Next these Taylor expansions are inserted into the discretized modal field equation

$$
L_{1} \psi_{i-1, j}+L_{2} \psi_{l, j}+L_{3} \psi_{i+1, j}+(\Delta x)^{2}\left[\delta_{y}^{2}+k_{0}^{2} n_{i, j}^{2}-n_{e}^{2}\right] \psi_{i, j}=0
$$

at the grid point $(i, j)$, using continuity relations (i)-(iii). In Equation $16, \delta_{y}^{2}$ denotes the discretized second-order derivative with respect to $y$. We also make use of the fact that $\partial^{2} \psi_{l} / \partial x^{2}=\partial^{2} \psi_{i, j} / \partial x^{2}+O(\Delta x)$. The result is

$$
L_{2}=-2+(\Delta x)^{2} k_{0}^{2}\left(\frac{1}{2}\left(n_{r}^{2}-n_{l}^{2}\right)+\epsilon\left(n_{l}^{2}-n_{r}^{2}\right)+\frac{\epsilon^{2}}{2}\left(n_{r}^{2}-n_{l}^{2}\right)\right)
$$

and $L_{1}=L_{3}=1$. Analogously for $(i+1, j)$, the coefficients $R_{1}, R_{2}, R_{3}$ in the second derivative

$$
\frac{\partial^{2} \psi_{i+1, j}}{\partial x^{2}}=\frac{R_{1} \psi_{i, j}+R_{2} \psi_{i+1, j}+R_{3} \psi_{i+2, j}}{(\Delta x)^{2}}+O(\Delta x)
$$

can be obtained by replacing $\epsilon$ by $1-\epsilon$ and by interchanging $n_{r}$ and $n_{l}$ in Equation 17:

$$
R_{2}=-2+(\Delta x)^{2} k_{0}^{2}\left(\frac{1}{2}\left(n_{l}^{2}-n_{r}^{2}\right)+(1-\epsilon)\left(n_{r}^{2}-n_{l}^{2}\right)+\frac{(1-\epsilon)^{2}}{2}\left(n_{l}^{2}-n_{r}^{2}\right)\right)
$$

and $R_{1}=R_{3}=1$. This result is exactly the same as found in [8] for the TE case in two dimensions.

Case $B: \psi$ perpendicular to interface

Now, (i) $n^{2} \psi$ is continuous, $K \psi_{l}=\psi_{r}$, with $K=n_{l}^{2} / n_{r}^{2}$ in Equation 15; (ii) $\partial \psi / \partial x$ is continuous, $(\partial \psi / \partial x)_{t}=(\partial \psi / \partial x)_{r}$; (iii) analogously to Case A, $\left(\partial^{2} \psi / \partial x^{2}\right)_{r}=K\left[\left(\partial^{2} \psi / \partial x^{2}\right)_{l}+\right.$ $\left.k_{0}^{2}\left(n_{l}^{2}-n_{r}^{2}\right) \psi_{l}\right]$.

Owing to the discontinuity of the field at the interface, $L_{1}, L_{3} R_{1}$ and $R_{3}$ also have to be adapted to achieve satisfaction of the continuity relations (i)-(iii). With help of the Taylor 
expansions (15) we have

$$
\begin{aligned}
& L_{3}=\frac{2}{2(K-1) \epsilon^{2}+(-K+1) \epsilon+K+1} \\
& L_{1}=[(K-1) \epsilon+1] L_{3} \\
& L_{2}=-[(K-1) \epsilon+K+1] L_{3}-\frac{K}{2}\left[k_{0} \Delta x(1-\epsilon)\right]^{2}\left(n_{l}^{2}-n_{r}^{2}\right) L_{3}
\end{aligned}
$$

For the grid point $\left(i+1, j\right.$ ) we can immediately write down the expressions for $R_{1}, R_{2}, R_{3}$, by interchanging the role of $L_{1}$ and $L_{3}$, replacing $\epsilon$ by $1-\epsilon$, replacing $K$ by $1 / K$ and interchanging $n_{r}$ and $n_{l}$ in Equations 20 to 22 :

$$
\begin{aligned}
& R_{1}=\frac{2}{2(1 / K-1)(1-\epsilon)^{2}+(-1 / K+1)(1-\epsilon)+1 / K+1} \\
& R_{3}=[(1 / K-1)(1-\epsilon)+1] R_{1} \\
& R_{2}=-[(1 / K-1)(1-\epsilon)+1 / K+1] R_{1}-\frac{1}{2 K}\left[k_{0} \Delta x \epsilon\right]^{2}\left(n_{r}^{2}-n_{l}^{2}\right) R_{1}
\end{aligned}
$$

For absorbing structures a problem can occur if the denominator in the expressions for $L_{1}, \ldots, R_{3}$ becomes zero. We shall restrict ourselves to the nonabsorbing case.

\subsection{Zero-order EICs}

In this section the zero-order EICs derived by Stern [9] and by Kim and Ramaswamy [10] are briefly recalled.

In the approach of [10], the cross-section consists of grid points $(i, j)$ located at the centre of rectangular cells having widths $\Delta x_{i, j}$ and $\Delta y_{i, j}$ in $x$ and $y$, respectively. The interface is located at the border of two adjacent cells. In this approach, the location of the interface with respect to the grid points can be varied by varying the widths of the cells, in other words by using a nonuniform grid. In our approach, we use a uniform grid, but the interface can still have a general position with respect to the grid points. The approach of [10] in terms of a uniform grid would be as follows.

Suppose the normal of the interface is parallel to $x$. Let the interface have distance $\epsilon \Delta x$ $(0 \leq \epsilon<1)$ to grid point $(i, j)$ and distance $(1-\epsilon) \Delta x$ to grid point $(i+1, j)$. In terms of our formulation,

$$
\Delta x_{l-1, J}=\Delta x_{i+1, J}=2(1-\epsilon) \Delta x \quad \Delta x_{l, j}=2 \epsilon \Delta x
$$

Case A: $\psi$ perpendicular to interface

Inserting the relations (26) into equation (10a) of [10] yields

$$
\begin{aligned}
\frac{\partial^{2} \psi_{l, j}}{\partial x^{2}}= & \frac{1}{2 \epsilon(\Delta x)^{2}}\left[\psi_{i-1, j}+\frac{n_{r}^{2}}{\epsilon n_{l}^{2}+(1-\epsilon) n_{r}^{2}} \psi_{l+1, j}-\left(1+\frac{n_{l}^{2}}{\epsilon n_{l}^{2}+(1-\epsilon) n_{r}^{2}}\right) \psi_{i, j}\right] \\
& +O\left((\Delta x)^{0}\right)
\end{aligned}
$$

whereas equation $(10 \mathrm{~b})$ of $[10]$ becomes

$$
\frac{\partial^{2} \psi_{i, j}}{\partial y^{2}}=\frac{\psi_{i, j-1}-2 \psi_{i, j}+\psi_{i, j+1}}{(\Delta y)^{2}}
$$


It is interesting to see that if the interface is put in the middle between the grid points (i.e. $\epsilon=1 / 2$ ), then Equation 27 takes the form

$$
\frac{\partial^{2} \psi_{i, j}}{\partial x^{2}}=\frac{1}{(\Delta x)^{2}}\left[\psi_{l-1, j}+\frac{2 n_{r}^{2}}{n_{l}^{2}+n_{r}^{2}} \psi_{i+1, J}-\left(1+\frac{2 n_{l}^{2}}{n_{l}^{2}+n_{r}^{2}}\right) \psi_{l, J}\right]+O\left((\Delta x)^{0}\right)
$$

which is exactly the result (A10a) of Stern [9].

Case B: $\psi$ parallel to interface

The finite-difference expression for both lateral second derivatives takes the standard form (as in Equation 28).

As the formulation of [9] is a special case of the formulation of [10], our first-order EICs, derived in Section 3.1, are compared in the next section with the EICs derived in [10], in particular with Equations 27 and 28.

\section{Results}

In this section our first-order EICs are compared to the EICs (27) and (28). The two EICs are tested for the fundamental quasi-TE mode. The mode is determined by launching an approximate mode calculated with the effective-index method [25], and then using the mode finding algorithm described in Section 2.2. Both formulations of the EICs (zero order and first order) have been implemented, and the rate of convergence for the effective index as a function of the lateral grid spacing $\Delta x$ and $\Delta y$ is studied.

In our example, the structure consists of a film of InP with rectangular cross-section of width $a=2 b=1.0 \mu \mathrm{m}$ in $\boldsymbol{x}$ and $\boldsymbol{y}$ directions, respectively, refractive index $n_{f}=3.2$, surrounded by air $\left(n_{s}=1.0\right)$, for a wavelength $\lambda=1.55 \mu \mathrm{m}$. The geometry of the cross-section is shown in Fig. 2.

Figure 3 shows the normalized effective index difference $\Delta B$ as a function of the square of the grid spacing $(\Delta x)^{2}=(\Delta y)^{2}$, with $\Delta B \equiv B-B_{\text {lim }}$ being defined as

$$
B=\frac{n_{e}^{2}-n_{s}^{2}}{n_{f}^{2}-n_{s}^{2}} \quad B_{11 \mathrm{~m}}=\lim _{\Delta x . \Delta y \rightarrow 0} B
$$
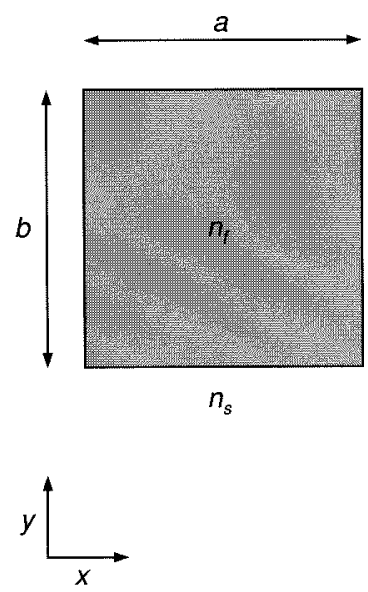

Figure 2 Geometry of the example described in the text. A rectangular film, having width $a, b$ In $\boldsymbol{x}$ - and $\boldsymbol{y}$-directions, respectively, with refractive index $n_{f}$, is surrounded by a substrate with refractive index $n_{s}$. 


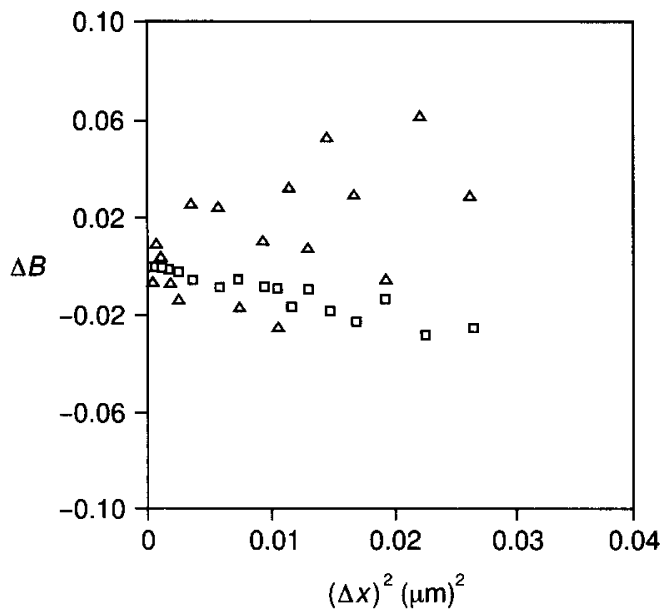

Figure 3 Normalized effective index difference $\Delta B$ of the fundamental quasi-TE mode as a function of the square of the grid spacing $(\Delta x)^{2}=(\Delta y)^{2}$, for both first-order $(\square)$ and zero-order $(\Delta)$ EICs.

for both EICs. We have taken $B_{\text {lim }}=0.791$ by extrapolating $B$ linearly as a function of $(\Delta x)^{2}$ to its value for $\Delta x=0$. The mode profile is depicted in Fig. 4 for one particular case of the data for first-order EICs in Fig. 3. To show that for the quasi-TE mode a discontinuity does indeed appear at the interfaces parallel to the $y$-axis, in Fig. 5 the field is plotted for a cross-section for constant $y=y_{M}$ (Fig. 5a) and for constant $x=x_{M}$ (Fig. 5b), where $\left(x_{M}, y_{M}\right)$ denotes the centre of the computational window.

From Fig. 3 it becomes clear that $\Delta B$ converges to zero for both zero-order and first-order EICs. The convergence for the first-order EICs is faster than that for the zero-order EICs. It can also be seen from Fig. 3 that there are fluctuations in the convergence, especially for the zeroorder EICs. Inspection of the EIC equations (27), (28) (zero-order) and (17) to (25) (first-order)

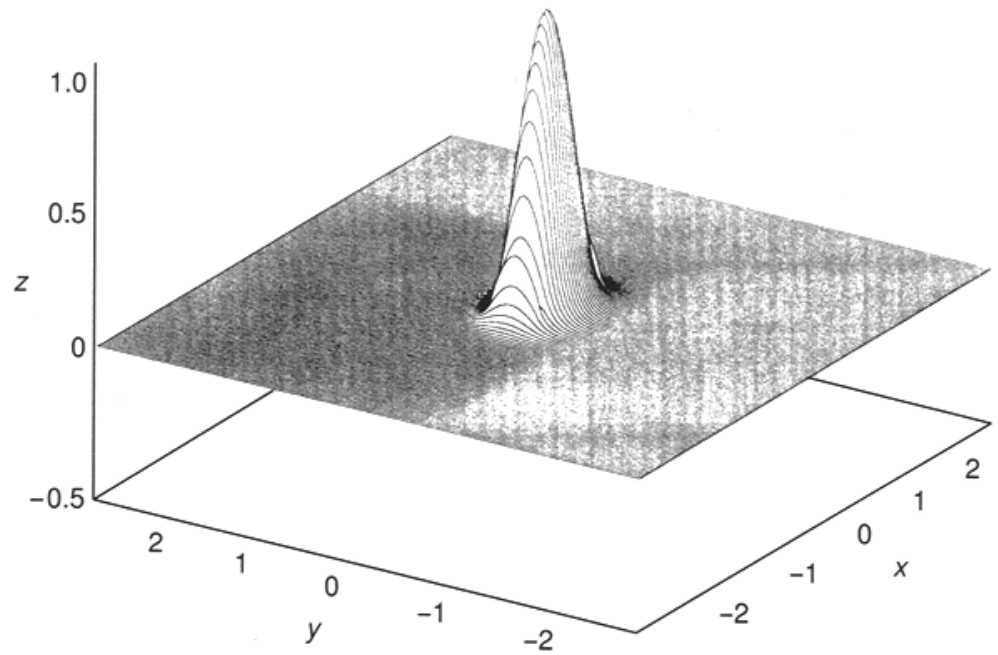

Figure 4 Fundamental quasi-TE mode for one of the data of Fig. 3, using first-order EICs, with $\Delta x=$ $\Delta y=0.046131 \mu \mathrm{m}$, and number of grid points $N_{x}=N_{y}=128$. 


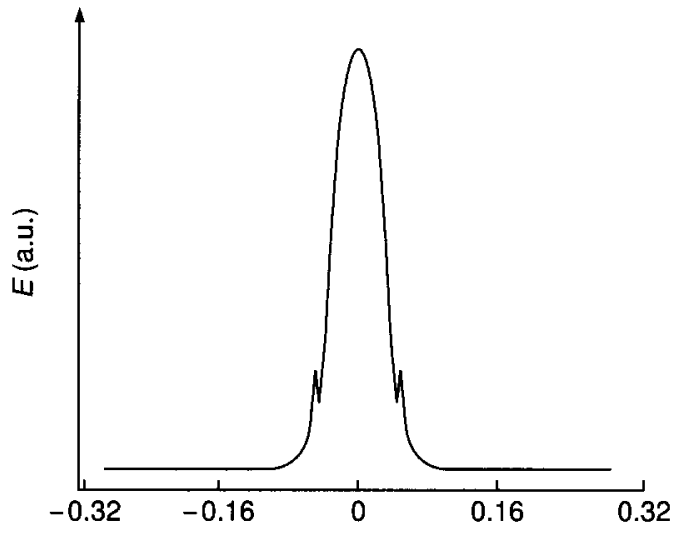

(a)

$10 x(\mu \mathrm{m})$

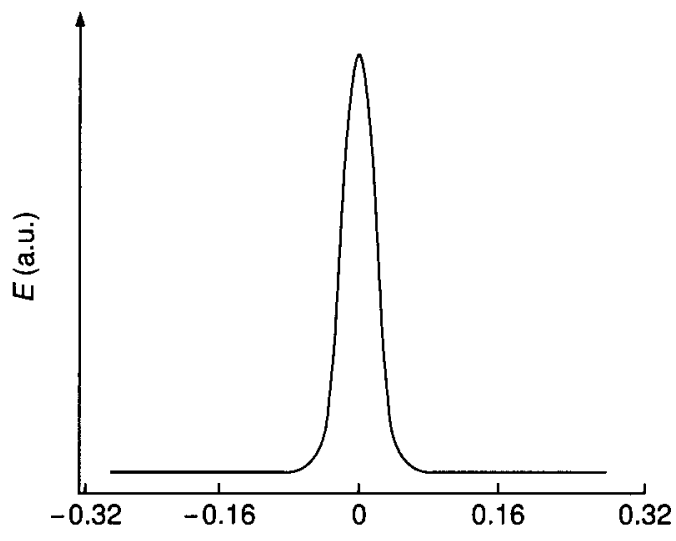

(b)

$10 x(\mu \mathrm{m})$
Figure 5 One-dimensional cross-section of the field shown in Fig. 4, for constant $y=y_{M}$ (a) and for constant $x=x_{M}$ (b); $\left(x_{M}, y_{M}\right)$ denotes the centre of the computational window.

suggests that the fluctuations might be caused by the fact that the value of $\epsilon$ will vary with variation of the grid spacing. A good test, therefore, would be a calculation with the interfaces midway between the grid points (i.e. $\epsilon=1 / 2$ ). Notice that the zero-order EICs of [10] in that case reduce to the expressions derived in [9].

Figure 6 shows $|\Delta B|$ as function of $(\Delta x)^{2}=(\Delta y)^{2}$ on a doubly logarithmic scale, with the geometry chosen such that both the interfaces parallel to $\boldsymbol{x}$ and $\boldsymbol{y}$ are midway between the grid points, for both EICs. For all data in Fig. 6, $|\Delta B|=+\Delta B(-\Delta B)$ for the zero- (first-) order EICs, respectively.

Figure 6 shows that the fluctuations in $\Delta B$ disappear, that $B$ depends more or less linearly on $(\Delta x)^{2}=(\Delta y)^{2}$, and that the first-order EICs give faster convergence than the zero-order EICs.

In order to study separately the dependence of $B$ on $\Delta x$ and on $\Delta y$, Fig. 7 gives $\Delta B$ as a function of $(\Delta x)^{2}$ for both EICs, with the discretization such that the interfaces parallel to the $y$-axis are in the middle between the grid points, with constant $\Delta y=0.046131 \mu \mathrm{m}$ and $N_{y}=128$.

In Fig. $8,|\Delta B|$ is plotted as a function of $(\Delta y)^{2}$ on a doubly logarithmic scale, for both EICs, with the discretization such that the interfaces parallel to the $x$-axis are midway between the grid points, and for constant $\Delta x=0.046131 \mu \mathrm{m}$ and $N_{x}=128$. It is seen from Figs 7 and 8 


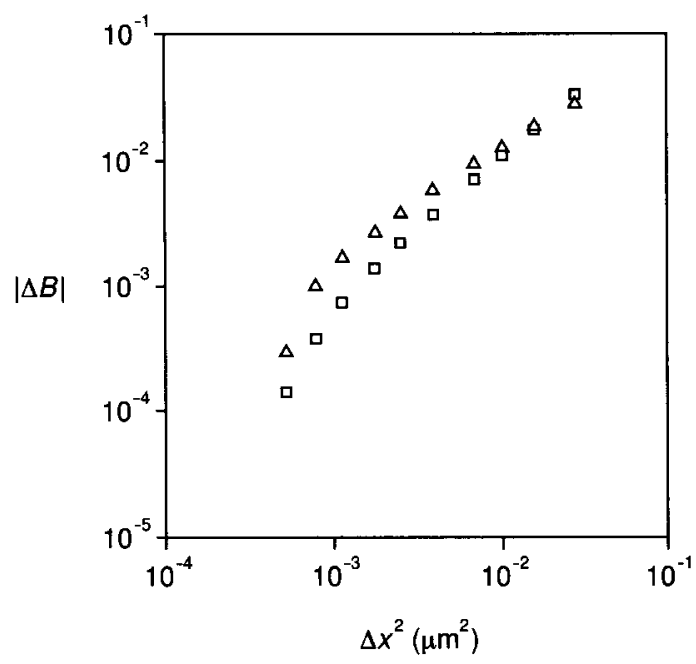

Figure 6 Normalized effective index difference $|\Delta B|$ of the fundamental quasi-TE mode as a function of the square of the grid spacing $(\Delta x)^{2}=(\Delta y)^{2}$, on a doubly logarithmic scale, with the geometry chosen such that the interfaces parallel to $x$ and $y$ are all midway between the grid points, for both first-order $(\square)$ and zero-order $(\Delta)$ EICs.

that varying $\Delta y$ has a larger influence than varying $\Delta x$. The reason is that the aspect ratio of the waveguide is $a / b=2 / 1$. Hence the field intensities near the right and left interfaces are smaller than the field intensities near the upper and lower interfaces, and consequently the variation of $\Delta y$ at the upper and lower interfaces will have a larger influence on the value of $B$ than the variation of $\Delta x$ at the right and left interfaces. For this reason, $\Delta B$ converges to a value significantly smaller than zero as $\Delta x \rightarrow 0$ in Fig. 7 , since $B$ depends strongly on the value of $\Delta y$, which is constant. On the other hand, $\Delta B$ converges to a value close to zero as $\Delta y \rightarrow 0$ in Fig. 8. Since $B$ does not depend strongly on the value of $\Delta x$, the fact that $\Delta x$ is constant does not have a large influence on the limiting value of $B$ as $\Delta y \rightarrow 0$.

Comparison of Figs 3 and 6 strongly suggests that the rate of convergence depends on the position of the interface with respect to the grid points.

For constant geometry in the $y$-direction such that the interface parallel to the $x$-axis is midway between the grid points, Fig. 9 shows $\Delta B$ as a function of $\epsilon_{x}$ defined at the interface on the

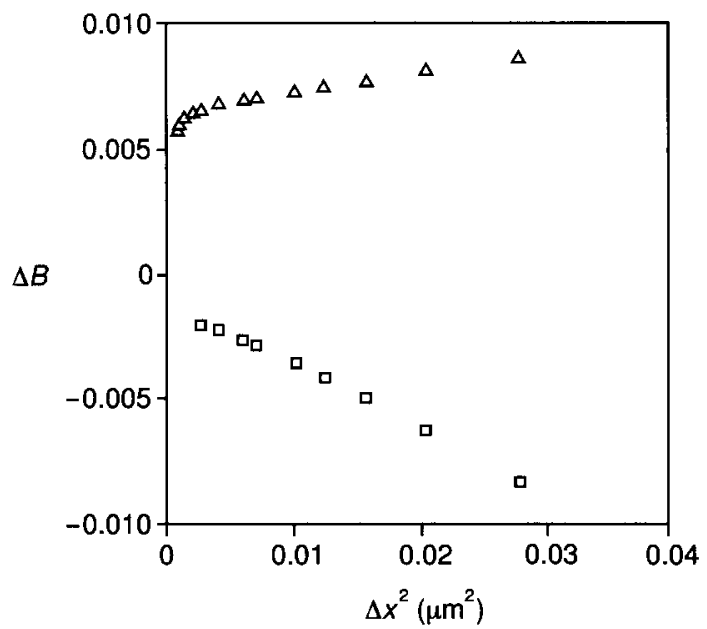

Figure 7 Normalized effective index difference $\Delta B$ of the fundamental quasi-TE mode as a function of the square of the grid spacing $(\Delta x)^{2}$, for constant discretization in the $y$-direction, $\Delta y=0.046131 \mu \mathrm{m}, N_{y}=128$, for both first-order $(\square)$ and zero-order $(\Delta)$ EICs. The discretization is such that the interfaces parallel to the $y$-axis are midway between the grid points. 


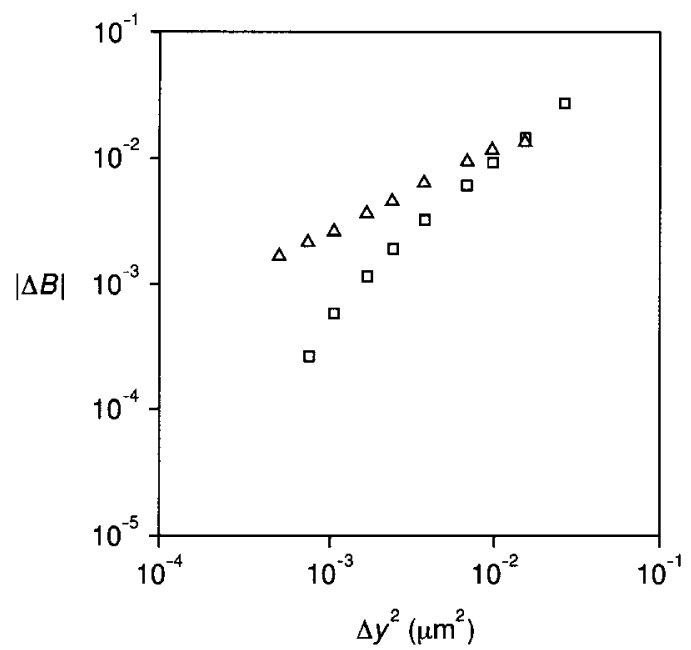

Figure 8 Normalized effective index difference $|\Delta B|$ of the fundamental quasi-TE mode as a function of the square of the grid spacing $(\Delta y)^{2}$, on a doubly logarithmic scale, for constant discretization in the $x$-direction, $\Delta x=0.046131 \mu \mathrm{m}, N_{y}=128$, for both firstorder $(\square)$ and zero-order $(\triangle)$ EICs. The discretization is such that the interfaces parallel to the $x$-axis are midway between the grid points.

left-hand side of the waveguide (see Figs 1 and 3). Here $\Delta x=\Delta y=0.022727 \mu \mathrm{m}$ and $N_{x}=N_{y}=256$. For constant geometry in the $x$-direction such that the interface parallel to the $y$-axis is midway between the grid points, Fig. 10 shows $\Delta B$ as a function of $\epsilon_{y}$ defined at the interface on the bottom side of the waveguide. Again $\Delta x=\Delta y=0.022727 \mu \mathrm{m}$ and $N_{x}=N_{y}=256$.

In the calculations on which Figs 9 and 10 are based, the geometry is such that the widths $a$ and $b$ of the waveguide are both multiples of the grid spacing. Consequently the results for $\epsilon_{x}$ and $1-\epsilon_{x}$ are identical in Fig. 9, and the results for $\epsilon_{y}$ and $1-\epsilon_{y}$ are identical in Fig. 10.

Figure 9 shows that the convergence of $B$ depends on the position of the left and right interfaces with respect to the grid points. This dependence is stronger for the zero-order EICs than for the first-order EICs. It is seen in Fig. 10 that there is no $\epsilon_{y}$-dependence for the zero-order EICs. The reason is that at interfaces parallel to the polarization (in this case along $x$ ), the finite-difference second derivatives do not depend on $\epsilon$ (Case B in Section 3.2).

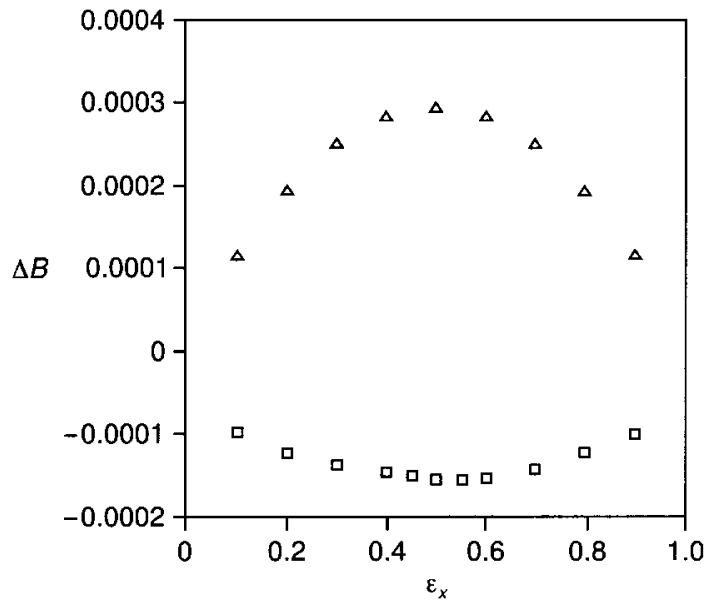

Figure 9 Normalızed effective index difference $\Delta B$ of the fundamental quasi-TE mode as a function of $\epsilon_{x}$ defined at the interface on the left-hand side of the waveguide (see Figs 1 and 3) for $\Delta x=\Delta y=0.022727 \mu \mathrm{m}, N_{x}=$ $N_{y}=256$, for both first-order ( $\square$ ) and zeroorder $(\Delta)$ EICs. 


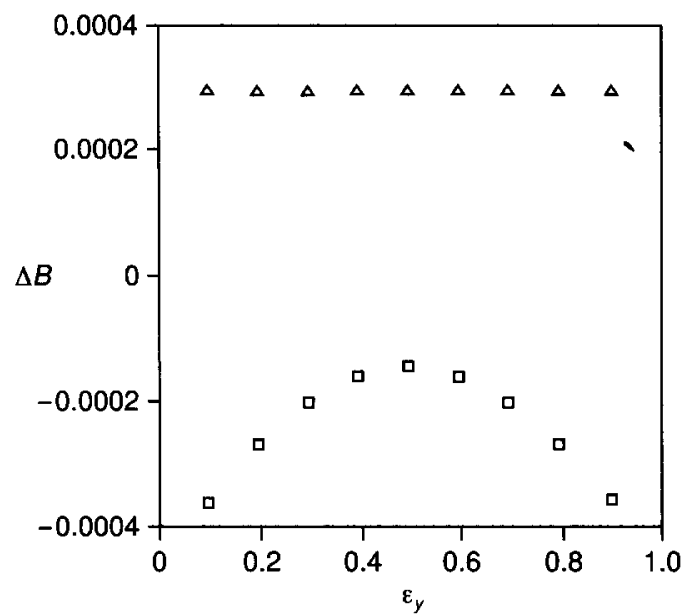

Figure 10 Normalized effective index difference $\Delta B$ of the fundamental quasi-TE mode as a function of $\epsilon_{y}$ defined at the interface on the bottom side of the waveguide for $\Delta x=\Delta y=0.022727 \mu \mathrm{m}, N_{x}=N_{y}=256$, for both first-order $(\square)$ and zero-order $(\triangle)$ ElCs.

For the first-order EICs, comparison of Figs 9 and 10 shows that the $\epsilon$-dependence is larger at the lower and upper interfaces than at the left and right interfaces. The reason is that the field intensities are much larger at the lower and upper interfaces than at the left and right interfaces due to the geometry of the waveguide. Consequently, in the former case $B$ is much more sensitive to variations in $\epsilon$ than in the latter case.

Comparing the zero-order and first-order EICs in terms of computer time, roughly the same number of iterations are needed to get convergence to a modal solution for both EICs. Also, the computer time per iteration is about the same, so in that sense there is no preference for either of the EICs.

Vassallo [11, 12] derived EICs by making a Taylor expansion for the discretized electric field as well. For the field parallel to the interface, the EICs are of second order, which is one order more accurate than our EICs. The extra accuracy stems from changing $L_{1}=L_{3}=1$ (see Section 3.1) to

$$
\begin{aligned}
& L_{1}=1+\frac{(\Delta x)^{2} k_{0}^{2}}{12}(1+2 \epsilon)(1-\epsilon)^{2}\left(n_{l}^{2}-n_{r}^{2}\right) \\
& L_{3}=1-\frac{(\Delta x)^{2} k_{0}^{2}}{12}(1+2 \epsilon)(1-\epsilon)^{2}\left(n_{l}^{2}-n_{r}^{2}\right)
\end{aligned}
$$

For the field perpendicular to the interface, the coefficients are exactly the same as in our case, so that then the EICs are of first order. For a calculation on a rib waveguide, Vassallo reports strong dependence of the effective index on $\epsilon_{x}$ [12] if the corners of the waveguide are not treated in a special way. The difference between the two formulations is the extra order of accuracy for the case with the field parallel to the interface. In our case, however, the EICs have been applied to the case of a rectangular cross-section, whereas Vassallo treated the case of a rib waveguide. To compare the two methods rigorously, one should apply both of them to the same geometry.

\section{Conclusions}

First-order EICs have been derived for the semivectorial FDBPM in the SVEA in order to achieve accuracy up to second order in the lateral grid spacing, leading to a first-order 
method. These EICs have been compared to zero-order EICs derived by Kim and Ramaswamy [10] and by Stern [9]. The zero- and first-order EICs yield convergence for the effective index to the same value when decreasing the lateral grid spacing, the rate of convergence being faster for the first-order EICs. Fluctuations are noticed in the convergence, especially for the zeroorder EICs; these have been shown to be caused by the variation in the interface position relative to the grid points, given by the parameter $\epsilon$. If the interface is put midway between grid points, these fluctuations disappear. Also, the first-order EICs then give faster convergence than the zero-order EICs.

The influence of the grid spacing at the two different interfaces depends strongly on the local field intensity, for both the zero-order and the first-order EICs.

By shifting the waveguide relative to a constant discretized grid in the two lateral directions separately, it has been shown that at interfaces perpendicular to the polarization the rate of convergence for the effective index depends on the position of the interface with respect to the grid points (given by $\epsilon$ ) for both EICs. This dependence is larger for the zero-order EICs than for the first-order EICs. At interfaces parallel to the polarization, there is no dependence on the interface position for the zero-order EICs.

For the first-order EICs, for the two lateral directions, the influence of the interface position on the effective index value, depends strongly on the local field intensities.

Our first-order EICs are almost identical to EICs derived by Vassallo [11, 12], who derived second- (first-) order EICs for the electric field parallel (perpendicular) to the interface. Vassallo noted a strong dependence of the effective index on the position of the interface with respect to the grid points [12]. In order to compare the two methods rigorously, both formulations on the EICs should be applied to the same geometry.

\section{Acknowledgements}

It is a pleasure to thank Ravi Varshney for stimulating discussions and one of the referees for drawing our attention to two papers by C. Vassallo deriving almost identical efficient interface conditions.

\section{References}

1. J. VAN ROEY, J. VAN DER DONK and P. E. LAGASSE, J. Opt. Soc. Am. 71 (1981) 423.

2. L. THYLEN, Opt. Quantum Electron. 15 (1983) 433.

3. P. E. LAGASSE and R. BAETS, Radio Sci. 22 (1987) 1225.

4. D. YEVICK and B. HERMANSSON, J. Quantum Electron. 26 (1990) 109.

5. Y. CHUNG and N. DAGLI, IEEE J. Quantum Electron. 26 (1990) 1335.

6. R. ACCORNERO, M. ARTIGLIA, G. COPPA, P. DI VITA, G. LAPENTA, M. POTENZA and P. RAVETTO, Electron. Lett. 26 (1990) 1959.

7. T. RASMUSSEN, J. H. POVLSEN and A. BJARKLEV, IEEE Photon. Technol. Lett. 5 (1993) 339.

8. H. J. W. M. HOEKSTRA, G. J. M. KRIJNEN and P. V. LAMBECK, J. Lightwave Technol. 10 (1992) 1352.

9. M. S. STERN, IEE Proc. J. 135(1) (1988) 56.

10. C. M. KIM and R. V. RAMASWAMY, J. Lightwave Technol. 7 (1989) 1581.

11. C. VASSALlO, IEE Proc. J. 139 (1992) 137.

12. C. VASSALlO, Proc. ECOC 1992, Berlin, paper We P2.25.

13. D. YEVICK and B. HERMANSSON, J. Quantum Electron. 25 (1989) 221.

14. J. C. CHEN and S. JÜNGLING, Opt. Quantum Electron. 26 (1994) S199.

15. J. C. CHEN and S. JUNGLING, Proc. IPR, 1994, paper ThF7-1.

16. S. JUNGLING and J. C. CHEN, IEEE J. Quantum Electron. 30 (1994) 2098.

17. F. WIJNANDS, H J. W. M. HOEKSTRA, G. J. M. KRIJNEN and R. M. DE RIDDER, J. Lightwave Technol. 12 (1994) 2066. 
18. F. WIJNANDS, H. J. W. M. HOEKSTRA, G. J. M. KRIJNEN and R. M. DE RIDDER, Proc. Linear and Nonlinear Integrated Optics, Lindau, 1994. SPIE 2212 (1994) 85.

19. F. WIJNANDS, H. J. W. M. HOEKSTRA, G. J. M. KRIJNEN and R. M. DE RIDDER, J. Quantum Electron. 31 (1995) 782.

20. A. R. MITCHELL and D. F. GRIFFITHS, The Finite Difference Method in Partial Differential Equations (Wiley, Chichester, 1980) p. 59.

21. W. H. PRESS, B. P. FLANNERY, S. A. TEUKOLSY and W. T. VETTERLING, Numerical Recipes (Cambridge University Press, Cambridge, 1986) p. 377.

22. P.-L. LIU, S. L. YANG and D. M. YUAN, IEEE J. Quantum Electron. 29 (1993) 1205.

23. G. R. HADLEY, Opt. Lett. 16 (1991) 624.

24. A. RALSTON and P. RABINOWITZ, A First Course in Numerical Analysis, 2nd edn (McGraw-Hill, New York, 1978) p. 498.

25. G. B. HOCKER and W. K. BURNS, Appl. Opt. 16 (1977) 113. 